\title{
The effect of intrathecal curcumin on mechanical allodynia in rats after L5 spinal nerve ligation
}

\author{
Ju Hwan Lee, Yeon Dong Kim, Hoe Chang Jung, and Yong Kwan Cheong \\ Department of Anesthesiology and Pain Medicine, Wonkwang University School of Medicine, Iksan, Korea
}

Curcumin is found in the yellow spice turmeric made from the roots (rhizomes) of Curcuma longa. Curcumin has been known to have various antinociceptive activities in some animal models, including an antinociceptive activity on neuropathic pain.

The anti-nociceptive effect of curcumin is exerted through its action on dorsal root ganglia, by inhibiting astrocyte activation and suppressing the expression of glial fibrillary acid protein after spinal cord injury [1]. Curcumin can further suppress astrocyte activation by inhibiting the secretion of tumor necrosis factor a (TNFa), interleukin (IL)-1 $\alpha$, IL-6, and nitric oxide.

This research was designed to examine whether the intrathecal injection of curcumin exerts an alleviating effect on mechanical allodynia in the rat spinal nerve ligation (SNL) model.

The approval for the experimental protocol was obtained from the Institutional Animal Care and Use Committee, Wonkwang University. The ligation of the L5 spinal nerve was performed in adult Sprague-Dawley rats (250-300 g). Sevoflurane was used to anesthetize the animals by delivery through a nose cone. A 6-0 silk suture was used to ligate the left L5 spinal nerve, which was cut distally.

Each animal was kept in a separate cage in a temperature controlled room $\left(23.0 \pm 0.5^{\circ} \mathrm{C}\right)$ with a $12 / 12$-hr light/dark cycle. Five days after nerve ligation, intrathecal catheterization was carried out in order to enable drug injection. Group $1(n=5)$ was the sham-operated group, group $2(n=5)$ underwent SNL but did not receive curcumin, whereas group $3(n=5)$ underwent SNL and then received $200 \mu$ g curcumin.

Curcumin solution was prepared in $100 \%$ dimethyl sulfoxide (DMSO). In the animals of groups 1 and 2, $10 \mu \mathrm{l}$ of $100 \%$ DMSO was injected intrathecally before injecting $10 \mu \mathrm{l}$ of normal saline; whereas in the animals of group 3, $200 \mu \mathrm{g}$ curcumin was injected intrathecally before injecting $10 \mu \mathrm{l}$ of normal saline.

The evaluation of the behavioral tests with curcumin administration was conducted on the 7th, 8th, 9th, 10th, 15th and 20th day after SNL. Measurement of paw withdrawal thresholds (PWTs) was made at 30 minutes after treatment. The drug was administered once a day from the 7 th day through the 20th day after SNL.

The area where mechanical allodynia was induced for the tests was the region between the foot pads on the plantar surface of the hind paw. The up-down method was employed for the quantification of the response to mechanical stimuli, while a set of von Frey filaments (Touch Test ${ }^{\circledR}$, North Coast Medical Inc., Gilroy, CA, USA) was used to apply forces of approximately logarithmic increments, so as to induce mechanical allodynia for the determination of mechanical PWTs.

Data are presented as mean \pm SEM. SPSS version 13.0 (SPSS Inc., Chicago, IL, USA) was used for statistical analysis. Comparison of data between different time points in the same group was made using the one-way repeated-measures ANOVA, while comparison of data between different groups was made using the two-way ANOVA. Comparison between specific behavioral data points in ANOVA was made by means of the Tukey honestly significant difference test for post-hoc analysis. Data from the tests were considered statistically significant only when the $\mathrm{P}$ value was $<0.05$.

Group 1, the sham-operated group, showed no change in ipsilateral PWTs. In contrast, groups 2 and 3, which had undergone SNL, showed a statistically significant decrease in ipsilat-

Corresponding author: Yong Kwan Cheong, M.D., Ph.D., Department of Anesthesiology and Pain Medicine, Wonkwang University School of Medicine, 460, Iksandae-ro, Iksan 570-749, Korea. Tel: 82-63-859-1560, Fax: 82-63-857-5472, E-mail: ykfolder@wonkwang.ac.kr

(c) This is an open-access article distributed under the terms of the Creative Commons Attribution Non-Commercial License (http:// creativecommons.org/licenses/by-nc/3.0/), which permits unrestricted non-commercial use, distribution, and reproduction in any medium, provided the original work is properly cited. 


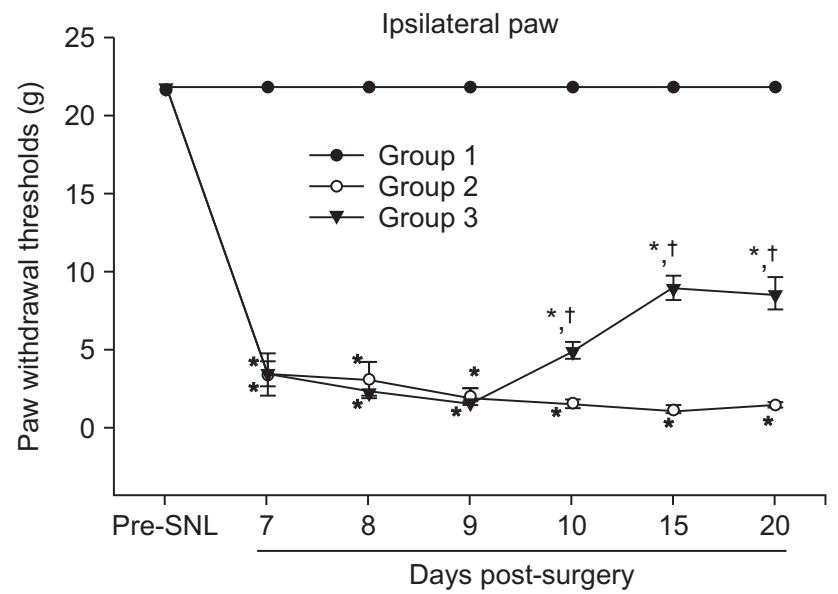

Fig. 1. The alleviating effect of intrathecally injected curcumin on mechanical allodynia in nerve-injured rats. Group $1(\mathrm{n}=5)$ is the shamoperated group, group $2(n=5)$ is the SNL group, and group $3(n=5)$ is the SNL with curcumin group. Groups 2 and 3 showed a statistically significant decrease in ipsilateral PWTs compared to group 1. Group 3 showed statistically significant increases in ipsilateral PWTs than group 2 on the 10th, 15th, and 20th day after surgery. $* \mathrm{P}<0.05$ compared to Group $1,{ }^{\dagger} \mathrm{P}<0.05$ compared to Group 2. SNL: spinal nerve ligation, PWTs: paw withdrawal thresholds.

eral PWTs compared to group $1(\mathrm{P}<0.05)$. When group 2 was compared with group 3 , there was no statistically significant difference in ipsilateral PWTs on the 7th, 8th, and 9th day after SNL. On the 10th, 15th, and 20th day after SNL, however, group 3 showed statistically significantly higher ipsilateral PWTs than group $2(\mathrm{P}<0.05)$ (Fig. 1).

There was no significant change in contralateral PWTs after treatment (Data not presented).

Preceding preclinical researches have shown that alteration in pain behavior was related to the elevation of TNF- $\alpha$ levels. TNF- $\alpha$ interacts with TNF- $\alpha$ receptor 1 to activate nuclear factor (NF)- $\mathrm{KB}$, consequently inducing the transcription of genes of inflammatory and other mediators of pain, including TNF- $\alpha$, IL 6 , and cyclooxygenase 2 .

Intrathecally delivered recombinant TNF- $\alpha$ induced mechanical allodynia and thermal hyperalgesia in rats; while intrathecally administered etanercept, a TNF- $\alpha$ inhibitor, could alleviate inflammatory pain. In addition, spinal synaptic plasticity could be effectively regulated by the interaction between TNF- $\alpha$ and transient receptor potential subtype V1 [2].

In the present research, groups 3 showed no significant change in PWTs on the 7th day through the 9th day after SNL in spite of curcumin injection compared to group 2, but only from the 10th day, group 3 showed a significant change in PWTs compared to group 2. The reason for this occurrence could not be ascertained. However, Zhu et el. [3] reported that significant anti-nociceptive effect could be observed after at least 5 days of daily curcumin administration during the time courses of thermal latency and mechanical threshold. In addition, Zhao et al. [4] reported that chronic, but not acute, curcumin treatment attenuated mechanical allodynia and thermal hyperalgesia in neuropathic mice.

Zhao et el. [4] reported that the levels of spinal monoamine (serotonin) and metabolite (3-methoxy-4-hydroxyphenylglycol, MHPG) were elevated by chronic curcumin treatment, while the modality-specific analgesic effect of curcumin disappeared completely after spinal noradrenaline or serotonin transmission was interrupted.

According to a research conducted by Han et al. [5] using a rat model that was created to show biphasic flinching response by the subcutaneous injection of formalin into the hind paw, intrathecal administration of curcumin resulted in reduction in the number of flinching responses in both phase 1 and phase 2 .

This research included intrathecal administration of $200 \mathrm{ug}$ curcumin only. Follow-up research that includes various doses and other methods of administration is necessary. In addition, future research on the efficacy of curcumin should include more diverse neuropathic pain models.

In conclusion, this research demonstrated the alleviating effect of intrathecally administered curcumin on mechanical allodynia in nerve-injured rats, and an additional research is necessary for assessing the spinal mechanism of action of curcumin.

\section{Acknowledgments}

This paper was supported by Wonkwang University in 2012.

\section{References}

1. Lin MS, Lee YH, Chiu WT, Hung KS. Curcumin provides neuroprotection after spinal cord injury. J Surg Res 2011; 166: 280-9.

2. Park CK, Lu N, Xu ZZ, Liu T, Serhan CN, Ji RR. Resolving TRPV1- and TNF- $\alpha$-mediated spinal cord synaptic plasticity and inflammatory pain with neuroprotectin D1. J Neurosci 2011; 31: 15072-85.

3. Zhu X, Li Q, Chang R, Yang D, Song Z, Guo Q, et al. Curcumin alleviates neuropathic pain by inhibiting p300/CBP histone acetyltransferase activity-regulated expression of BDNF and cox-2 in a rat model. PloS One 2014; 9: e91303.

4. Zhao X, Xu Y, Zhao Q, Chen CR, Liu AM, Huang ZL. Curcumin exerts antinociceptive effects in a mouse model of neuropathic pain: Descending monoamine system and opioid receptors are differentially involved. Neuropharmacology 2012; 62: 843-54.

5. Han YK, Lee SH, Jeong HJ, Kim MS, Yoon MH, Kim WM. Analgesic effects of intrathecal curcumin in the rat formalin test. Korean J Pain 2012; 25: 1-6. 